J. Clin. Chem. Clin. Biochem.

Vol. 18, 1980, pp. 897-899

\title{
Wirkung von Rifampicin und Streptomycin auf die Konzentrationen von Testosteron und Cortisol im Blut von Männern
}

\author{
Von Lieselotte Nocke-Finck, H. Breuer
}

Institut für Klinische Biochemie der Universität Bonn und

\section{Reimers}

Lungenkrankenhaus Bethanien, Solingen-Aufderhöhe

(Eingegangen am 25. April 1980)

Zusammenfassung: In früheren Untersuchungen ist gezeigt worden, daß Rifampicin die Biosynthese und den Stoffwechsel von Östrogenen beeinflußt. Um festzustellen, ob Rifampicin auch auf die Konzentrationen von Testosteron und Cortisol eine Wirkung ausïbt, wurden bei fünf tuberkulosekranken Männern unter Therapie mit Rifampicin oder Streptomycin entsprechende Hormonbestimmungen durchgeführt. Unter Behandlung mit Rifampicin waren die Testosteronkonzentrationen um $8 \mathrm{Uhr}$ signifikant höher als vor der Behandlung. Auch bei den Blutentnahmen um 16.30 Uhr waren die Werte bis auf eine Ausnahme signifikant erhöht. Unter Streptomycintherapie wurden bei zwei Patienten ein Anstieg, bei einem Patienten ein Abfall und bei zwei Patienten keine Veränderungen gegenüber der Zeit vor der Behandlung gefunden. Für Cortisol konnten während der Rifampicin-Behandlung bei zwei Patienten signifikante Erhöhungen der Plasmawerte und bei drei Patienten keine Veränderungen festgestellt werden. Die Cortisolwerte änderten sich in keinem Fall während der Streptomy cin-Therapie.

Aus den vorliegenden Ergebnissen kann der Schluß gezogen werden, daß Rifampicin - im Gegensatz zu Streptomycin - die Biosynthese von Testosteron und möglicherweise auch Cortisol beeinflußt.

\section{Effects of rifampicin and streptomycin on the concentrations of testosterone and cortisol in the blood of men}

Summary: It has been shown previously that the biosynthesis and metabolism of oestrogens is affected by rifampicin. The present study was undertaken to investigate if rifampicin may also influence the plasma concentrations of testosterone and cortisol. Five male patients suffering from tuberculosis received rifampicin or streptomycin. During treatment with rifampicin testosterone concentrations were significantly elevated as compared to control conditions; this increase was found in the morning $(8.00 \mathrm{~h})$ as well as in the afternoon $(16.30 \mathrm{~h})$. When the same patients were treated with streptomycin, only irregular changes of testosterone concentrations were observed. The blood level of cortisol was increased in two patients, but unchanged in three patients who were treated with rifampicin. No changes at all were detected in patients under treatment with streptomycin. The results presented here show that rifampicin - in contrast to streptomycin - influences the biosynthesis of testosterone and possibly also that of cortisol.

\section{Einführung}

Im Jahre 1973 haben wir erstmalig über die Wirkung von Rifampicin auf den Menstruationszyklus und die Östrogenausscheidung bei Einnahme oraler Kontrazeptiva berichtet $(1,2)$. Ferner wurde nach Inkubation von $17 \alpha-$ Ethinylöstradiol-17 $\beta$ mit Lebermikrosomen von Frauen, die mit Rifampicin behandelt worden waren, eine vierfach verstärkte Hydroxylierung an Ring A und B des Ste- roids beobachtet (3). Nach oraler Gabe von radioaktivmarkiertem Mestranol (3-Methylether von 17 $\alpha$-Ethinylöstradiol-17 $\beta$ ) an Frauen, die gleichzeitig Rifampicin erhielten, war die Ausscheidung von $17 \alpha$-Ethinylöstradiol$17 \beta$ als Folge einer verstärkten Hydroxylierung am aromatischen Ring A vermindert (4).

In der vorliegenden Arbeit wurden die Plasmakonzentrationen von Testosteron und Cortisol vor und nach Gabe 
von Rifampicin und Streptomycin untersucht, um weitere Hinweise über die Wirkung von Antibiotika auf den Steroidstoffwechsel zu erhalten.

\section{Patienten und Methodik}

Fünf Patienten im Alter von 27 bis 51 Jahren wurden wegen einer Lungentuberkulose stationär behandelt.

Die Patienten erhielten zunächst eine Therapie von 400-500 $\mathrm{mg} / \mathrm{d}$ Isoniazid (Neoteben ${ }^{\circledR}$ ) und $1500-1900 \mathrm{mg} / \mathrm{d}$ Ethambutol (Myambutol ${ }^{\circledR}$ ); nach mindestens 10 Tagen dieser Therapie wurde an weiteren 5 Tagen unter dieser Therapie jeweils um 8 Uhr und um 16.30 Uhr Blut entnommen.

Anschließend erhielten die selben Patienten zusätzlich zu Isoniazid und Ethambutol $1 \mathrm{~g} / \mathrm{d}$ Streptomycin; nach mindestens 10 Tagen dieses Therapieschemas wurden an weiteren 5 Tagen unter dieser Therapie jeweils um $8 \mathrm{Uhr}$ und um 16.30 Uhr Blutproben entnommen.

Schließlich wurde Streptomycin durch 600-750 mg/d Rifampicin (Rifa ${ }^{\circledR}$ ) ersetzt und Blut nach dem gleichen Schema wie beschrieben entnommen.

Das heparinisierte Blut wurde sofort nach der Blutentnahme zentrifugiert und das Plasma bei $-20^{\circ} \mathrm{C}$ eingefroren.

Die radioimmunologische Bestimmung von Testosteron erfolgte nach der Methode von Nieschlag \& Loriaux (5). Cortisol wurde mit der Proteinbindungsmethode nach Nugent \&Mayes (7) gemessen. Alle Bestimmungen wurden in Triplikaten durchgefuihrt. Die statistischen Berechnungen erfolgten mit dem Test nach Student.

\section{Ergebnisse}

Das Verhalten von Testosteron im Blut von fünf Männern ist in Abbildung 1 und Abbildung 2 dargestellt. Abbildung 1 zeigt die Testosteronwerte nach Blutentnahme um $8 \mathrm{Uhr}$; jede Säule gibt den Mittelwert aus jeweils einer

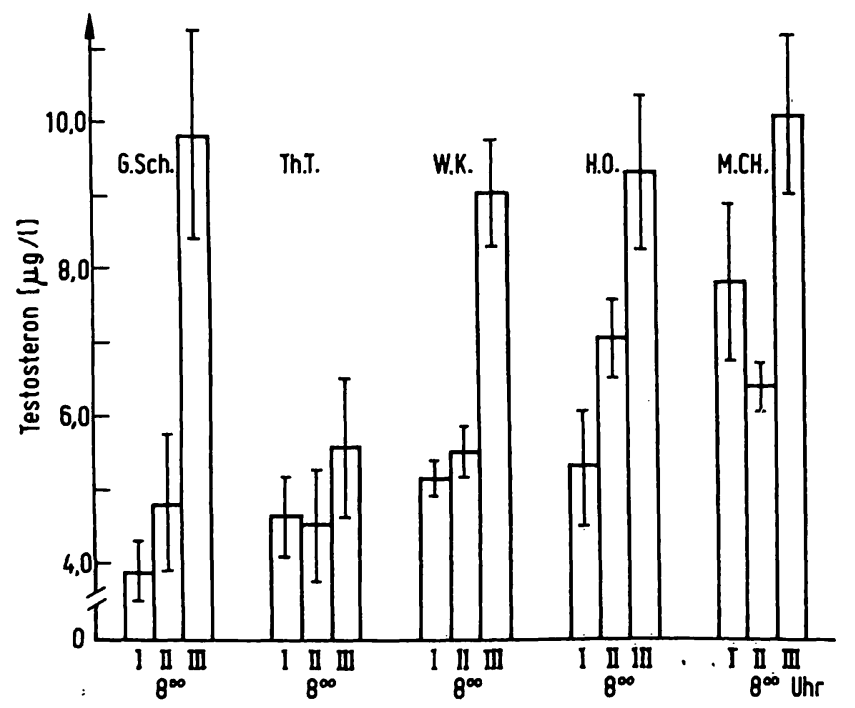

Abb. 1. Konzentrationen von Testosteron im Blut von fünf tuberkulosekranken Männern; Zeitpunkt der Blutentnahme 8 Uhr.

Behandlungsschema: I = Isoniazid und Ethambutol, II = Isoniazid, Ethambutol und Streptomyçin, III = Isoniazid, Ethambutol und Rifampicin. Weitere Einzelheiten siehe Text.

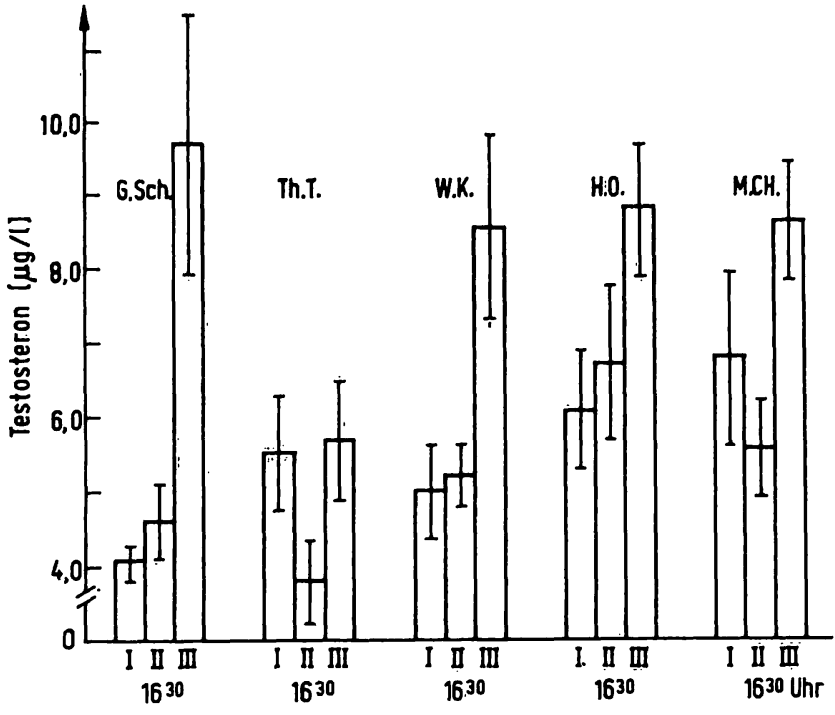

Abb. 2. Konzentrationen von Testosteron im Blut von fünf tuberkulosekranken Männern; Zeitpunkt der Blutentnahme 16.30 Uhr.

Behandlungsschema: I = Isoniazid und Ethambutol, II = Isoniazid, Ethambutol und Streptomycin, III = Isoniazid, Ethambutol und Rifampicin.

Blutentnahme an fünf aufeinanderfolgenden Tagen an. Die Verabreichung von Streptomycin führte bei zwei Patienten (G. Sch., H. O.) zu einer signifikanten Erhöhung von Testosteron. Bei zwei weiteren Patienten (Th. T., W. K.) wurde keine Veränderung festgestellt, bei einem Patienten (M. Ch.) war die Konzentration von Testosteron signifikant erniedrigt. Unter Behandlung mit Rifampicin ergab sich bei allen fünf Patienten ein signifikanter Anstieg von Testosteron $(p=0,005$ bis $p=0,0005)$. Bei den Blutentnahmen um 16.30 Uhr war unter Streptomycin kein signifikanter Anstieg von Testosteron zu beobachten (Abb. 2). Unter Behandlung mit Rifampicin erfolgte bei vier Patienten ein signifikanter Anstieg ( $p=$ 0,005 und $p=0,01$ bei M. Ch.) von Testosteron. Bei einem Patienten ergaben sich keine Veränderungen.

In Tabelle 1 sind die Ergebnisse der Cortisolbestimmungen wiedergegeben. Bei keinem der fün Patienten war unter Streptomycin eine Erhöhung der Cortisolkonzentrationen im Blut zu finden, wohl aber eine Emiedrigung in einem Falle (Th. T.).

Auch unter der Behandlung mit Rifampicin traten bei drei Patienten keine Veränderungen der Cortisolwerte auf. Allerdings waren bei zwei Patienten (W. K., M. Ch.) signifikante Erhöhungen zu beiden Blutentnahmezeiten zu beobachten.

\section{Diskussion}

Die vorliegenden Untersuchungen zeigen; daß unabhängig vom Zeitpunkt der Blütentnahme die Testosteronkonzentrationen im Blut tuberkulosekranker Männer unter Behandlung mit Rifampicin gegenüber den Kontrollbe- 
Tab. 1. Konzentrationen von Cortisol im Blut von fünf tuberkolosekranken Männern; Zeitpunkte der Blutentnahmen $8 \mathrm{Uhr}$ und 16.30 Uhr.

Behandlungsschema I, II und III siehe Legenden zu den Abbildungen. Die p-Werte wurden für die Werte bei Behandlung nach Schema II und III gegen die nach Schema I berechnet. $n$. s. = nicht signifikant.

\begin{tabular}{llllll}
\hline Patient & $\begin{array}{l}\text { Behand- } \\
\text { lungs- } \\
\text { schema }\end{array}$ & $\begin{array}{l}\text { 8 Uhr } \\
\text { Cortisol } \\
\mu \mathrm{g} / \mathrm{l}\end{array}$ & $\mathrm{p}$ & \multicolumn{3}{c}{$\begin{array}{l}\text { 16.30 Uhr } \\
\text { Cortisol } \\
\mu \mathrm{g} / \mathrm{l}\end{array}$} & $\mathrm{p}$ \\
\hline G. Sch. & I & 176 & - & 107 & - \\
& II & 145 & n.s. & 108 & n.s. \\
& III & 167 & n.s. & 114 & n.s. \\
Th. T. & I & 184 & - & 50 & - \\
& II & 77 & 0,01 & 42 & n. s. \\
& III & 121 & n.s. & 71 & n.s. \\
W. K. & I & 62 & - & 24 & - \\
& II & 67 & n.s. & 35 & n.s. \\
& III & 100 & 0,005 & 57 & 0,001 \\
H. O. & I & 189 & - & 97 & - \\
& II & 180 & n. s. & 141 & n. s. \\
& III & 209 & n.s. & 125 & n.s. \\
M. Ch. & I & 135 & - & 52 & - \\
& II & 132 & n.s. & 60 & n.s. \\
& III & 200 & 0,025 & 72 & 0,005 \\
\hline
\end{tabular}

dingungen deutlich erhöht sind. Im Unterschied dazu hat Streptomycin keine eindeutige Wirkung auf die Testosteronwerte. Die Erhöhung der Testosteronwerte bei rifampicinbehandelten Männern könnte die Folge einer veränderten Biosynthese oder eines verminderten Stoffwechsels sein. Im ersteren Fall wäre an eine Aktivierung, im zweiten Fall an eine Hemmung der beteiligten Enzyme zu denken. Nun ist bekannt, daß Rifampicin vornehmlich eine induzierende, zumindest aktivierende Wirkung auf Enzyme des endoplasmatischen Reticulums ausübt. So ist die Beobachtung, daß Rifampicin die Wirksamkeit oraler Kontrazeptiva beeinträchtigen kann, auf einen beschleunigten Abbau von 17 $\alpha$-Ethinylöstradiol$17 \beta$ zurückzuführen; dieses ist seinerseits durch eine vermehrte Hydroxylierung vorwiegend am C-Atom 2 bedingt. Diese Ergebnisse legen die Vermutung nahe, daß die Erhöhung von Testosteron im Blut rifampicinbehandelter Männer ebenfalls die Folge einer gesteigerten $\mathrm{Hy}$ droxylaseaktivität ist. Bei dem an der Biosynthese von Testosteron entscheidend beteiligten Enzym handelt es sich um die $17 \alpha$-Hydroxylase, die von Cytochrom P 450 abhängig ist (7).

In der Tat konnte in vorläufigen Versuchen mit der Mikrosomenfraktion von Rattentestes gezeigt werden, daß eine Behandlung der Tiere mit Rifampicin eine erhöhte Aktivität der $17 \alpha$-Hydroxylase und damit eine vermehrte Bildung von Testosteron aus $17 \alpha$-Hydroxyprogesteron auslöst (8). Dieser Befund ist kompatibel mit der Beobachtung von Remmer (9), wonach Rifampicin das für die Wirksamkeit von Hydroxylasen erforderliche Cytochrom $P 450$ in der Leber induziert.

Im Gegensatz zu Testosteron verhielten sich Cortisolwerte im Plasma rifampicinbehandelter Männer uneinheitlich. Nur bei zwei Patienten waren - unabhängig von der circadianen Rhythmik - Anstiege zu beobachten. In diesem Falle ist es schwierig zu entscheiden, ob und in welchem Umfang Rifampicin die an der Biosynthese und am Stoffwechsel beteiligten Enzyme beeinflußt. Versuche mit geeigneten Enzympräparationen sind notwendig, um den möglichen Einfluß von Rifampicin zu charakterisieren.

\section{Literatur}

1. Nocke-Finck, L., Breuer, H. \& Reimers, D. (1973), Acta Endocrinol. (Kbh.) Suppl. 177, 136.

2. Nocke-Finck, L., Breuer, H. \& Reimers, D. (1973), Dtsch. Med. Wochenschr. 98, 1521-1523.

3. Bolt, H. M., Kappus, H. \& Bolt, M. (1975), Europ. J. Clin. Pharmacol. 8, 301-307.

4. Gelbke, H. P., Gethmann, U. \& Knuppen, R. (1977), Horm. Metab. Res. 9, 415-419.

5. Nieschlag, E. \& Loriaux, D. L. (1972), Z. Klin. Chem. 10, $164-168$.

6. Nugent, Ch. A. \& Mayes, D. M. (1966), J. Clin. Endocrinol. 26, 1116-1122.

7. Loriaux, D. L., Menard, R., Taylor, A., Pita, J. C \& Sauten, R. (1976), Ann. Int. Med. 85, 630-633.

8. Nocke-Finck, L., Wipping, F. W. \& Breuer, H. (1979), Acta Endocrinol. (Kbh.) Suppl. 225, 90. Ausführliche Mitteilung: Acta Endocrinol. (Kbh.), im Druck.

9. Remmer, H., Schoene, B., Fleischmann, R. A. (1973), Drug Metab. Disposition 1, 224-230.

Professor Dr. H. Breuer Institut für Klinische Biochemie Sigmund-Freud-Straße 25 D-5300 Bonn 1 
